\title{
The dangers of the fast track
}

\begin{abstract}
The US Food and Drug Administration (FDA) wants to create a way to fast-track approval of new medical devices, but they must first address the problems of the existing accelerated process for bringing devices to market that are substantially equivalent to ones already in commercial distribution.
\end{abstract}

$\mathbf{T}$ he FDA is responsible for evaluating the safety and efficacy of medical devices, such as insulin pumps and cardiovascular stents, and deciding whether they should be approved for use in patients. However, a recent study found flaws in the fast-track $510(\mathrm{k})$ process the FDA uses to evaluate $99 \%$ of device submissions (Arch. Intern. Med. doi:10.1001/archinternmed.2011.30,2011). In spite of this, the FDA is currently proposing a new way to fast-track approval of novel devices http:/ go.nature.com/BPSxZQ). For the sake of patient safety, the FDA needs to fix its current accelerated device approval process before it considers adding anything new to it.

Medical devices currently reach patients by two routes: premarket approval (PMA) - which is given to a wholly new device and includes evaluation of clinical trial data to demonstrate safety and efficacy_or the $510(\mathrm{k})$ pathway for devices similar to ones already on the market. In the latter case, which was initially meant for devices that would pose low risk to patients if they were to malfunction, companies need merely to demonstrate relative equivalency of materials and purpose to an existing device, often with no clinical trial data necessary.

However, after the passage of the 2002 Medical Device User Fee and Modernization Act, which allowed the FDA to choose the "least burdensome approach" to evaluate devices, more and more truly new devices have been going through the $510(\mathrm{k})$ avenue, including 'high-risk' devices that could pose major health risks if they should prove defective. The FDA seems to have been forced to make this choice by their limited budget: the $510(\mathrm{k})$ process is cheaper for both the FDA $(\$ 18,200$ compared with $\$ 870,000$ for PMA) and for the company looking to sell the device (fee of $\$ 4,000$ compared with $\$ 218,000$ for PMA). Currently, only $1 \%$ of devices evaluated by the FDA go through the longer and costlier PMA process. This caused the US Government Accountability Office (GAO) to sound the alert in January 2009 of the potential danger to patients of this lower level of oversight.

Now, the Archives of Internal Medicine study demonstrates that the GAO may have been right: the study shows that of the 113 high-risk device recalls that occurred from 2005 to 2009, 71\% of them had come to market through the faster and cheaper $510(\mathrm{k})$ pathway. One such device is a cardiac defibrillator that was surgically implanted into 268,000 patients, causing 13 deaths when it malfunctioned. It has since been recalled. Although the study authors don't mention what percentage of the total number of devices approved by the FDA have been recalled, and device manufacturers argue that this percentage is extremely low, the findings of this study indicate that the $510(\mathrm{k})$ process should be reevaluated.

In August 2010, the FDA came out with a series of recommendations to improve the $510(\mathrm{k})$ process, including asking for more clinical trial data and postmarket studies. These recommendations would cost more both for the FDA, in terms of the additional personnel needed to analyze the data, and for medical device companies, who would need to conduct the extra studies and who oppose these proposed changes. It is currently unclear how many of these recommendations the FDA will be able to implement. Congress should act now to ensure that the FDA has the funding it needs so that these proposed changes can be put in place to ensure the safety of patients that use these devices.

In this environment of regulatory uncertainty, some device manufacturers are shutting their doors in the US and moving their operations overseas, where device approval is easier and less expensive. US patients must then go abroad if they need these devices. To address this issue of company and patient exodus from the US, the FDA is considering a new way to approve novel devices, in which it will collaborate with companies at early stages of development, even prior to the start of clinical trials. The idea is that increasing the communication between FDA decision makers and companies at an early stage of the process will reduce device development costs and speed the delivery of these new devices to the patients in need in as little as six months. The FDA cautions that, given their current resources, this could only be possible for one or two truly new devices per year.

The idea of enhancing communication between the FDA and device manufacturers at early stages of device development is an admirable goal, but the FDA must also focus its resources on the current problems with the $510(\mathrm{k})$ process. The FDA needs to consider whether all novel or high-risk devices should be evaluated and approved with clinical trial data via PMA, keeping the $510(\mathrm{k})$ process for low-risk devices, as it was originally intended. This may help the FDA fix what's broken in its device approval process so it can best ensure patient safety. 